\title{
When Does a Quantum Field Theory Describe Particles?^
}

\author{
By
}

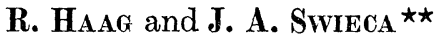 \\ Department of Physics, University of Illinois, Urbana, Illinois
}

\begin{abstract}
We give a criterion which has to be satisfied in a Quantum Field Theory in order to allow a complete particle interpretation of the theory. The notion of "essentially localized states" in Field Theory is re-examined.
\end{abstract}

\section{Introduction}

The usually accepted postulates of relativistic quantum field theory, namely Lorentz invariance, local commutativity, causality, and the structure of the energy-momentum spectrum are not sufficient to ensure an interpretation of physical states entirely in terms of asymptotic particle configurations. This fact is demonstrated by the well known example of a generalized free field with continuous weight function [1]. This model satisfies all the above requirements if the weight function is suitably chosen ${ }^{\star \star \star}$ yet it does not allow a complete particle interpretation.

It is the purpose of this paper to suggest a possible criterion that would distinguish between field theories with and without particle interpretation.

This criterion is a generalization to relativistic quantum field theories of the old quantum mechanical argument: the number of quantum states of a particle in a finite phase space volume $\Omega$ is finite, namely $\Omega / h^{3}$.

Pursuing for the moment an intuitive argument one would expect that in a theory with short range forces and particle interpretation a physical state which at time $t=0$ is essentially localized in a finite region of space and has limited energy will quickly evolve into a state described by a configuration of essentially non-interacting particles, finite in number and still localized in a finite space volume with finite energy.

* Supported in part by the National Science Foundation.

$\star \star$ On leave of absence from University of São Paulo, Brazil.

$\star \star \star$ Lorentz invariance, local commutativity and the energy momentum spectrum are immediately evident. "Primitive causality" is violated if the weight function does not decrease rapidly enough for large mass values [2] but is satisfied for models with a fast decreasing weight function [3]. 
Therefore, according to the earlier heuristic argument there should be only a finite number of linearly independent states which are localized at $t=0$ in any given finite space region and have energy below any given value $E_{\max }$.

Having thus put forth the heuristic basis for our criterion we will give its field theoretical formulation.

One of the difficulties that must be overcome is that in a relativistic field theory it is not possible to define a class of states strictly localized in a finite region of space within a given time interval if we want to keep all the properties which one would like to associate with the notion of localization, as it is familiar from non-relativistic quantum mechanics.

Specifically, if one wants to define the set of states which are localized in the 3-dimensional space volume $V$ during the time interval $t_{1}<t<t_{2}$ one might be tempted to identify it with the subspace $\mathscr{H}_{v, t_{1}, t_{2}}$ which is obtained by applying the algebra $R(\mathcal{O})$ [2], [4] associated with the space-time region

$$
\mathcal{O}: \mathbf{x} \in V ; \quad t_{1}<x_{0}<t_{2}
$$

on the vacuum state $|0\rangle$. This is, however, impossible because it turns out that $\mathscr{H}_{v, t_{1}, t_{2}}$ is not a subspace but the whole Hilbert space. This fact has first been demonstrated by SchLIEDER and REEH [5]. It has been suggested by KNIGHT [6] that one should not apply the whole algebra $R(\mathcal{O})$ but only its unitary operators to the vacuum in order to obtain localized states. This is a workable definition and very similar in fact to the one we shall adopt and try to justify in Section II. It means, however, that the "localized states" do not form a linear space.

If one takes the set of all "localized" states in a region $\mathcal{O}$ and projects out those with energy less than $E_{\max }$, one would expect, that in theories with short range forces and particle interpretation one obtains an "essentially" finite set of linearly independent vectors. More precisely one would expect that the resulting set of vectors contains only a finite number of orthogonal vectors with norm larger than an arbitrary given number. In other words, this set of vectors should be contained in a (strongly) compact subset of the Hilbert space.

We formulate the compactness criterion in Section III and show in Section IV that it is satisfied for the free field theory and is not satisfied for a generalized free field theory with continuous weight function, i.e., for the situation in which there exists no particle interpretation.

Thus, loosely speaking the criterion is satisfied if and only if a theory has the same "number of degrees of freedom" as a free field theory. However, we shall not in the present paper attempt to elaborate on this point and we cannot show here that the compactness criterion will always ensure a complete particle interpretation. 


\section{Localization of States}

Let $\mathcal{O}$ be a finite space-time region, $R(\mathcal{O})$ the associated ring of bounded operators, $|0\rangle$ the vacuum state. We consider the subset $S_{r}(\mathcal{O})$ of operators $Q$ with the properties

$$
\begin{gathered}
Q \in R(\mathcal{O}) \\
\langle 0|Q| 0\rangle=0 \\
\|Q\| \leqq e^{x r} \| Q|0\rangle \| ;
\end{gathered}
$$

and the corresponding subset of states

$$
\mathscr{M}_{r}(\mathcal{O})=S_{r}(\mathcal{O})|0\rangle .
$$

Here $x$ is the smallest mass in the theory and $r$ an arbitrary length. We show now that the states of $\mathscr{M}_{r}(\mathcal{O})$ have a number of properties which allow us to picture them intuitively as being essentially localized in the region $\mathcal{O}+x$ with $|\mathbf{x}|<r, x_{0}=0$.

The first of these properties is the following. Let $\mathcal{O}_{1}$ and $\mathcal{O}_{2}$ be two regions which are situated space-like to each other and let us use as a measure of their separation the largest time-displacement $\tau$ which can be applied in positive and negative directions to the region $\mathcal{O}_{2}$ without any of its points entering into a time-like situation to some point of $\mathcal{O}_{1}$. Then, if $\Psi \in \mathscr{M}_{r}\left(\mathcal{O}_{2}\right)$ and $\Phi \in \mathscr{N}_{r}\left(\mathcal{O}_{1}\right)$

$$
|\langle\Phi, \Psi\rangle|<2\|\Phi\|\|\Psi\| e^{2 \varkappa r} \varphi(\varkappa \tau)
$$

where $\varphi$ is a function which decreases exponentially for large argument (see estimate below). The estimate (3) shows that the two states are very nearly orthogonal if $\tau-2 r$ is larger than a few Compton lengths.

To prove (3) we may use a method developed by RUELLE [7] and Araki [4]. Let $f^{\prime}(\omega)$ be an infinitely differentiable function with support in the interval $-x<\omega<x$, and

Define

$$
\int f^{\prime}(\omega) d \omega=1 .
$$

$$
\begin{gathered}
f(\omega)=\int_{-\infty}^{\omega} f^{\prime}\left(\omega^{\prime}\right) d \omega^{\prime}, \\
f_{\gamma}(t)=(2 \pi)^{-1} \int f(\omega) e^{-i \omega t-\gamma \omega} d \omega \\
f(t)=\lim _{\gamma \rightarrow 0} f_{\gamma}(t) .
\end{gathered}
$$

Note that $f(\omega)$ is infinitely often differentiable everywhere, it vanishes for $\omega<-\varkappa$ and has the constant value 1 for $\omega>\varkappa$. For $t \neq 0$ we find

$$
f(t)=(2 \pi i t)^{-1} \int f^{\prime}(\omega) e^{-i \omega t} d \omega .
$$

It is immediately seen therefore, that $|f(t)|$ decreases faster than any power of $t$. Now, if $Q$ is any operator with $\langle 0|Q| 0\rangle=0$, let $Q(t)$ be its 
time translate and $Q(f)=\int Q(t) f(t) d t$. We have, due to the gap of size $x$ in the energy spectrum,

$$
Q(f)|0\rangle=Q|0\rangle ; \quad Q^{\dagger}(f)|0\rangle=0 .
$$

Therefore, if $Q_{1} \in S_{r}\left(\mathcal{O}_{1}\right)$ and $Q_{2} \in S_{r}\left(\mathcal{O}_{2}\right)$ we get

$$
\begin{aligned}
& =\left\langle 0\left|Q_{1}^{\dagger} Q_{2}\right| 0\right\rangle=\left\langle 0\left|\left[Q_{1}^{\dagger}, Q_{2}(f)\right]\right| 0\right\rangle \\
& =\int_{|t|>\tau}\left\langle 0\left[Q_{1}^{\dagger}, Q_{2}(t)\right] \mid 0\right\rangle f(t) d t \leqq 2\left\|Q_{1}\right\|\left\|Q_{2}\right\|_{|t|>\tau} f(t) d t .
\end{aligned}
$$

If $\Psi=Q_{1}|0\rangle, \Phi=Q_{2}|0\rangle$ and if the $Q_{i}$ satisfy (1), then the inequality (10) coincides with (3) if we define

$$
\varphi(\varkappa \tau)=\inf \left|\int_{|t|>\tau} f(t) d t\right|
$$

where the symbol inf means that we can choose for every $\tau$ that function $f$ within the class specified above which gives the smallest value of $\varphi$.

We shall not attempt here to get the optimal estimate for $\varphi$ but consider only a simple family of trial functions $f$ which suffices to show that $\varphi$ is exponentially decreasing. Choosing the length scale so that $x=1$ we use

$$
\begin{aligned}
f^{\prime}(\omega) & =N^{-1} \exp -C\left(1-\omega^{2}\right)^{-1} \text { for }-1 \leqq \omega \leqq 1 \\
N & =\int_{-1}^{1} \exp -C\left(1-\omega^{2}\right)^{-1} d \omega .
\end{aligned}
$$

We calculate $f(t)$ by equation (8) using the fact that the integrand is an analytic function of $\omega$ in the whole plane except for the essential singularities at the end points of integration. The integrand of (8) has two sharp maxima (saddle points) within the unit circle. Deforming the path of integration to pass over these saddle points and using the simple estimate

$$
N>(2 / C)^{1 / 2} \exp \left[\frac{C}{1-1 / 2 C}\right]
$$

for the normalization integral, one finds that the optimal choice of $C$ is $C=3 / 8 \sqrt{2} t$, yielding for $|t|>3$

Thus, for $\varkappa \tau>3$

$$
|f(t)|<\frac{1}{2|t|} \exp [-t / 2 \sqrt{2}]
$$

$$
\varphi(\varkappa \tau)<\exp [-\varkappa \tau / 2 \sqrt{2}] .
$$

For the simple family of functions (12) the estimate (15) is about optimal. But one would expect that it can be improved to

$$
\varphi(\varkappa \tau)<A \exp [-\varkappa \tau]
$$

by a better choice of $f$. 
Property (3) is not yet a sufficient criterion for localized states. If it were, we could replace (1) by the weaker requirement

$$
\| Q^{\dagger}|0\rangle\left\|<e^{\varkappa r}\right\| Q|0\rangle \|
$$

We must, however, also guarantee that a state $\Phi$ which is localized in $\mathcal{O}_{1}$ is almost orthogonal to any "multiply localized" state $\Psi$, even if one of the localization centers of $\Psi$ lies in $\mathcal{O}_{1}$. It suffices to consider a state of the form

$$
\Psi=Q_{1}^{\prime} Q_{2}|0\rangle
$$

with $Q_{1}^{\prime} \in S_{r}\left(\mathcal{O}_{1}\right), Q_{2} \in S_{r}\left(\mathcal{O}_{2}\right)$ where $\mathcal{O}_{2}$ has the separation $\tau$ (see above) from $\mathcal{O}_{1}{ }^{\star}$. In this case we get by the previous method

$$
\begin{gathered}
\langle\Phi, \Psi\rangle=\int_{|t|>\tau}\left\langle 0\left|\left[Q^{\dagger} Q_{1}^{\prime}, Q_{2}(t)\right]\right| 0\right\rangle f(t) d t \\
|\langle\Phi, \Psi\rangle|<2\left\|Q_{1}\right\|\left\|Q_{1}^{\prime}\right\|\left\|Q_{2}\right\| \varphi(x \tau) .
\end{gathered}
$$

Again we can replace $\left\|Q_{1}\right\|$ by $e^{\varkappa r}\|\Phi\|$ due to (1). Also

$$
\left\|Q_{1}^{\prime}\right\|\left\|Q_{2}\right\|<e^{x r^{\prime}}\|\Psi\|
$$

with $r^{\prime}=2 r$ if $\tau \gg r$. This follows from

i.e.

$$
\begin{aligned}
\langle\Psi, \Psi\rangle & =\left\langle 0\left|Q_{1}^{\dagger} Q_{1}^{\prime} Q_{2}^{\dagger} Q_{2}\right| 0\right\rangle=\left\langle 0\left|Q_{1}^{\dagger} Q_{1}^{\prime}\right| 0\right\rangle\left\langle 0\left|Q_{2}^{\dagger} Q_{2}\right| 0\right\rangle+ \\
& +\int_{|t|>\tau}\left\langle 0\left|\left[Q_{1}^{\prime \dagger} Q_{1}^{\prime}, Q_{2}^{\dagger}(t) Q_{2}(t)\right]\right| 0\right\rangle f(t) d t
\end{aligned}
$$

$$
\|\Psi\|^{2}>\left\|Q_{1}^{\prime}\right\|^{2}\left\|Q_{2}\right\|^{2}\left[e^{-4 x r}-\varphi(x \tau)\right],
$$

if $\tau$ is large enough, so that the first term in the bracket is bigger than the second. This is inequality (19) with

$$
e^{\varkappa r^{\prime}}=\left(e^{-4 \varkappa r}-\varphi(\varkappa \tau)\right)^{-1 / 2}
$$

or

$$
r^{\prime} \rightarrow 2 r \text { for large } \tau \text {. }
$$

The scalar product between the 2 -fold localized state $\Psi$ and the singly localized state $\Phi$ is then, due to (18)

$$
|\langle\Phi, \Psi\rangle|<2 e^{\varkappa\left(r+r^{\prime}\right)}\|\Phi\|\|\Psi\| \varphi(\varkappa \tau) .
$$

More generally, we say that a state $\Psi$ has $n$ localization centers, situated in the regions $\mathcal{O}_{i}(i=1 \ldots n)$ if

$$
\Psi=\prod_{1}^{n} Q_{i}|0\rangle ; \quad Q_{i} \in S_{r}\left(\mathcal{O}_{i}\right)
$$

and if the minimal separation $\tau$ of any two regions $\mathcal{O}_{i}$ is large compared to $r$ and $x^{-1}$. In that case we have

$$
\prod_{1}^{n}\left\|Q_{i}\right\|<A\|\Psi\|
$$

* Such a state will be called "doubly localized". 
with $A$ depending only on $r$ and $\tau$. If we take the scalar product of two such states whose localization centers are respectively in the regions $\mathcal{O}_{i}(i=1 \ldots m)$ and $\mathscr{O}_{j}^{\prime}(j=1 \ldots n)$ then we find

where

$$
|\langle\Phi, \Psi\rangle|<A^{\prime}\|\Phi\|\|\Psi\| \varphi(\varkappa \tau)
$$

$$
\tau=\sup _{j} \inf _{i} \tau_{i j}
$$

and $\tau_{i j}$ is the separation between $\mathcal{O}_{i}$ and $\mathcal{O}_{j}^{\prime}$.

There remain the following two questions:

1) Does the set $\mathscr{M}_{r}(\mathcal{O})$ contain essentially all localized states in $\mathcal{O}$ ?

2) How can the localized states be characterized, if instead of the ring of bounded operators $R(\mathcal{O})$ we want to work with the unbounded operators (Wightman polynomials) which result from "smeared out" fields ?

A proposal concerning the second question has recently been made by Schlieder [8]. We shall examine both questions together by using a criterion for localization which has first been proposed by KNIGHT and ToLL [6].

If $\Psi$ is localized in $\mathcal{O}$ and $Q \in R\left(\mathcal{O}_{1}\right)$ where $\mathcal{O}_{1}$ is (space-like) far separated from $\mathcal{O}$, then the expectation value of $Q$ in $\Psi$ should differ very little from the vacuum expectation value. We demand, if $\tau$ is the separation between $\mathcal{O}$ and $\mathcal{O}_{1}$ and if $\|\Psi\|=1$

$$
\langle\Psi \Psi|Q| \Psi\rangle-\langle 0|Q| 0\rangle \mid\langle 2 c\|Q\| \varphi(x \tau) .
$$

where $c$ is some fixed positive number. This criterion, demanded for arbitrary $\mathcal{O}_{1}$ and arbitrary $Q \in R\left(\mathcal{O}_{1}\right)$ should be necessary as well as sufficient for localization. Suppose now that

$$
\Psi=A|0\rangle
$$

where $A$ is a (possibly unbounded) operator associated with the ring $R(\mathcal{O})$. In particular $A$ could be a Wightman polynomial with test functions having support in $\mathcal{O}$. We denote the set of all operators which are associated with $R(\mathcal{O})$ by $\mathscr{P}(\mathcal{O})$. Note that the choice

$$
A \in \mathscr{P}(\mathcal{O})
$$

imposes essentially no restriction on $\Psi$ because of the theorem by Schlieder and REeH [5]. Since we want $\Psi$ to be normalized we have

$$
\left\langle 0\left|A^{\dagger} A\right| 0\right\rangle=1 \text {. }
$$

By the previously described method and the use of local commutativity the left hand side of (26) is brought into the form

$$
\int_{|t|>\tau}\left\langle 0\left|\left[A^{\dagger} A, Q(t)\right]\right| 0\right\rangle f(t) d t<2 \| A^{\dagger} A|0\rangle\|\| Q \| \varphi(\varkappa \tau) .
$$

Thus the criterion (26) requires

$$
\| A^{\dagger} A|0\rangle \|<c .
$$


If, as previously, we consider also states which are not normalized we are led to the set $\mathscr{N}_{c}(\mathcal{O})$ consisting of vectors $\Psi=A|0\rangle$ with

$$
\begin{gathered}
A \in \mathscr{P}(\mathcal{O}) \\
\| A^{\dagger} A|0\rangle\|<c\| \Psi \|^{2} .
\end{gathered}
$$

We shall show now that the set $\mathscr{N}_{c}(\mathcal{O})$ is simply related to the sets $\mathscr{H}_{r}(\mathcal{O})$. If we make a polar decomposition

$$
A=T H
$$

where $T$ is isometric, $H$ Hermitian and positive semi-definite, then

$$
A^{\dagger} A=H^{2}, \quad H \in \mathscr{P}(\mathcal{O}),
$$

and condition (29) becomes

$$
\left\langle 0\left|H^{4}\right| 0\right\rangle\left\langle c^{2}\left\langle 0\left|H^{2}\right| 0\right\rangle^{2} .\right.
$$

Suppose now, we replace $A$ by

$$
B=T P_{\lambda} H
$$

where $P_{\lambda}$ is the spectral projection operator of $H$ which annihilates the part of Hilbert space belonging to spectral values of $H$ greater than $\lambda$. Then $B$ is a bounded operator with

and we have

$$
\|B\|=\lambda
$$

$$
\left\langle 0\left|H^{4}\right| 0\right\rangle>\lambda^{2}\left\langle 0\left|\left(1-P_{\lambda}\right) H^{2}\right| 0\right\rangle=\lambda^{2} \|(A-B)|0\rangle \|^{2} .
$$

Thus, according to condition (32)

$$
\|(A-B)|0\rangle\left\|<\frac{c}{\lambda}\right\| \Psi \|^{2} .
$$

Putting $B|0\rangle=\Phi, \frac{\lambda}{\|\Phi\|}=e^{x r}$ we have

$$
\begin{gathered}
(\Phi-\langle 0, \Phi\rangle|0\rangle) \in \mathscr{M}_{r}(\mathcal{O}) \\
\frac{\|\Psi-\Phi\|}{\|\Psi\|}<2 c e^{-\varkappa r}
\end{gathered}
$$

for $e^{x r}>4 c$. Thus a state in $\mathscr{N}_{c}(\mathcal{O})$ can be approximated as

$$
\Psi=\gamma|0\rangle+\Psi^{\prime} ; \quad \Psi^{\prime} \in \mathscr{M}_{r}(\mathcal{O})
$$

up to a relative error of magnitude $2 c e^{-\varkappa r}$.

It is not so simple to compare the sets $\mathscr{M}_{r}(\mathcal{O})$ or $\mathscr{N}_{c}(\mathcal{O})$ to those sets which ScHLIEDER [8] defines as localized states in a Wightman Theory. In the first place, ScHLIEDER restricts simultaneously the momentum of the state and its space extension. But even when we apply an energy projection on $\mathscr{M}_{r}(\mathcal{O})$ the resulting set is still larger than the corresponding Schlieder set. The former is noncompact in the case of the generalized free field (section IV), the latter is compact [8]. We 
have tried to demonstrate above that every vector in $\mathscr{M}_{r}(\mathcal{O})$ can be interpreted as an essentially localized state. Therefore our present opinion is that the conditions imposed by Schlieder are sufficient but not always necessary for localization.

\section{The Compactness Criterion}

A state of energy less than $E$ can have at most $E / x$ particles. If it were strictly localized during some time interval in a 3-dimensional volume $V=r_{0}^{3}$ the phase volume would be roughly

$$
N\left(E, r_{0}\right)=\left(E r_{0}\right)^{3 E / \varkappa}
$$

and, with $\hbar=1$, this should again be the dimension of the subspace of $\mathscr{H}$ in which such states lie.

Our discussion in the last section shows that we are never dealing with strictly localized states, but that a state in $\mathscr{M}_{r}(\mathcal{O})$ has a probability of order $\varphi(x \tau)$ to extend beyond a sphere of radius $r_{0}+r+\tau$.

Consider now the set of vectors

$$
\Psi \in P_{E} \mathscr{M}_{r}(\mathcal{O}) ; \quad\|\Psi\|<1,
$$

where $P_{E}$ is the projection operator to energy values less than $E$. If $r_{0}$ or $r$ are not too small we may expect that the localization properties of the state are not significantly affected by the application of $P_{E}$. Actually there is some delocalization due to the energy projection. This can be minimized by using for $P_{E}$ not a sharp energy projection but a smooth energy "cut off". The following detailed estimates [e.g., eq. (44)] are true only for a smooth cut off but the compactness criteria will be relevant even for a sharp projection.

Because of the lack of strict localization the set (39) does not lie in a finite dimensional subspace. However we may define an approximate dimension in the following way. For any fixed $N$ we choose the "best" $N$-dimensional space $\mathscr{H}_{N}$ so that the maximal orthogonal distance of the vectors in (39) from $\mathscr{H}_{N}$ is as small as possible. This quantity, the thickness of the set (39) perpendicular do the best $\mathscr{H}_{N}$, will be denoted by $d_{N}$. As $N$ increases, $d_{N}$ decreases. From the argument above we get the implicit relation for the set (39);

$$
d_{N}=\varphi(\varkappa \tau) ; \quad N=N\left(E ; r_{0}+r+\tau\right) \approx\left[E\left(r_{0}+r+\tau\right)\right]^{3 E / \varkappa} .
$$

For $\tau \gg r+r_{0}$ and $\varphi$ given by $\left(15^{\prime}\right)$ we have

$$
d_{N} \approx \exp -\left[(x / E) N^{x / 3} E\right] \text {. }
$$

A very similar estimate can be made, if $\mathscr{M}_{r}$ in (39) is replaced by $\mathscr{N}_{c}$. Apart from numerical factors we only have to replace $r$ by $\varkappa^{-1} \log c$. Finally we may consider the set of vectors

$$
\Psi=P_{E} Q|0\rangle ; \text { with }\|Q\| \leqq 1, Q \in R(\mathcal{O})
$$


Again one finds a similar estimate for the thickness $d_{N}$ :

$$
d_{N} \approx 2 \exp -\left[x / 2 E N^{\varkappa / 3 E}\right] \text {. }
$$

The theory of approximate dimension, expressed in terms of the relation between $d_{N}$ and $N$ has been extensively studied in the mathematical literature $\star$. We are here concerned with bounded sets of vectors in Hilbert space for which $d_{N}$ decreases exponentially with some power of $N$. Such sets are compact in the norm topology. We summarize:

Criterion: In a Quantum Field Theory with minimal mass $x$ the sets (39), (42) should be compact in order to allow a particle interpretation. The thickness $d_{N}$ should decrease with $N$ like ${ }^{\star \star}$

$$
d_{N}<a e^{-\alpha N^{\beta}}
$$

where $\alpha$ and $\beta$ are of order $x / E$.

The same applies to the sets

and

$$
\Psi=P_{E} A|0\rangle, \text { with } \| A^{\dagger} A|0\rangle \|<1, A \in \mathscr{P}(\mathcal{O})
$$

$$
\Psi=P_{E} \mathscr{N}_{c}(\mathcal{O}) \text {, with }\|\Psi\|<1 \text {. }
$$

\section{Discussion of two Simple Models}

We show here that the criteria of the last section are satisfied in the theory of a scalar free field and that they are not satisfied in a generalized free field with continuous, fast decreasing weight function. Thus - at least in the simple models - the criteria serve to differentiate between a situation in which a particle interpretation is possible and one in which it is impossible. If suffices to investigate the compactness of the set (42) since the other three sets are simply related to $(42)$.

\section{a) The free-field $A(x)$}

Let us take for $\mathcal{O}$ the double cone which has a its base the 3-dimensional sphere of radius $r$ around the origin at $t=0$. We shall use the canonical creation and destruction operators at time zero which are denoted by $a^{\dagger}(\mathrm{x})$ and $a(\mathrm{x})$ respectively. They are related to the field by

$$
a(\mathrm{x})=\int\left[\Delta^{1 / 2}(\mathrm{x}-\mathrm{y}) A(\mathrm{y}, 0)+i \Delta^{-1 / 2}(\mathrm{x}-\mathrm{y}) \frac{\partial A}{\partial t}(\mathbf{y}, 0)\right] d^{3} y
$$

with the improper functions

$$
\Delta^{ \pm 1 / 2}(\mathbf{x})=\frac{1}{\sqrt{2}}(2 \pi)^{-3} \int\left(\mathbf{k}^{2}+m^{2}\right)^{ \pm 1 / 4} e^{i \mathbf{k x}} d^{3} k
$$

Consider a state vector

$$
\Psi=Q|0\rangle \text { with } Q \in R(\mathcal{O}), \quad\|Q\|<1,\langle 0|Q| 0\rangle=0 .
$$

* We are indebted to D. KastLer and M. ZERNER for an exposition of this subject. See e.g., [9], [10].

$\star \star$ This estimate is true only if $P_{E}$ is a smooth cut-off. 
We may express $\Psi$ in terms of its Newton-Wigner wave functions $f^{(n)}\left(\mathbf{x}_{1} \ldots \mathbf{x}_{n}\right)$ at time $t=0$.

$$
\begin{gathered}
\Psi=\int f^{(1)}(\mathrm{x}) a^{\dagger}(\mathrm{x}) d^{3} x+\frac{1}{\sqrt{2 !}} \int f^{(2)}\left(\mathrm{x}_{1}, \mathrm{x}_{2}\right) a^{\dagger}\left(\mathrm{x}_{1}\right) a^{\dagger}\left(\mathrm{x}_{2}\right) \times \\
\times d^{3} x_{1} d^{3} x_{2}+\cdots|0\rangle
\end{gathered}
$$

Choosing some radius $R \gg r$ we decompose the wave functions into

where

$$
f^{(n)}=g^{(n)}+h^{(n)}
$$

$$
g^{(n)}\left(\mathbf{x}_{\mathbf{1}} \ldots \mathbf{x}_{n}\right)=\prod_{i} \theta\left(R-\left|\mathbf{x}_{i}\right|\right) f^{(n)}\left(\mathbf{x}_{1} \ldots \mathbf{x}_{n}\right)
$$

vanishes if any argument $\mathbf{x}_{i}$ is outside of the sphere with radius $R$.

There are two main steps in the proof of the compactness criterion:

1) Show that

$$
\int\left|h^{(n)}\right|^{2} d^{3} x_{1} \ldots d^{3} x_{n}<A_{n} R^{4} e^{-2 m R}
$$

where $A_{n}$ is independent of the choice of $Q$.

2) Show that the operator in the $n$-particle subspace

$$
K_{E, R}=P_{E} \prod_{i} \theta\left(R-\left|\mathbf{x}_{i}\right|\right)
$$

is a compact operator.

The first statement is very similar to the results of section II and it may suffice here to sketch the proof for $f^{(1)}$. We have, for $|\mathbf{x}|>r+d$, neglecting numerical factors

$$
\begin{gathered}
f^{(\mathbf{1})}(\mathbf{x})=\langle 0|[a(\mathbf{x}), Q]| 0\rangle \\
=\left\langle 0\left|\int \Delta^{1 / 2}(\mathbf{x}-\mathbf{y})[A(\mathbf{y}, 0), Q] d^{3} y+\int \Delta^{-1 / 2}(\mathbf{x}-\mathbf{y})[\dot{A}(\mathbf{y}, 0), Q] d^{3} y\right| 0\right\rangle .
\end{gathered}
$$

Due to local commutativity we can replace the functions $\Delta^{ \pm 1 / 2}$ above by

$$
\sigma_{d}^{ \pm}(\mathbf{x}-\mathbf{y})=\Delta^{ \pm 1 / 2}(\mathbf{x}-\mathbf{y}) \tilde{\theta}(|\mathbf{x}-\mathbf{y}|-d)
$$

where $\tilde{\theta}$ is a function which has the constant value 1 for positive argument, vanishes for argument smaller than $-\varepsilon$ and rises in a smooth manner from 0 to 1 in the interval $[-\varepsilon, 0]$. The $\sigma_{\bar{d}}^{t}$ are smooth functions and one easily can get the following estimate for their Fourier transforms

Therefore the vectors

$$
\left|\tilde{\sigma}_{\bar{d}}^{ \pm}(\mathbf{k})\right|<\frac{C d}{\left(1+\left|\frac{\mathbf{k}}{m}\right|^{n}\right)} e^{-m d} .
$$

$\int \sigma_{d}^{(+)}(\mathbf{x}-\mathbf{y}) A(\mathbf{y}, 0) d^{3} y|0\rangle$ and $\int \sigma^{(-)}(\mathbf{x}-\mathbf{y}) A(\mathbf{y}, 0) d^{3} y|0\rangle$

are well defined and have norms smaller than $C^{\prime} d e^{-m d}$. This gives us the estimate

$$
\left|f^{(\mathbf{1})}(\mathbf{x})\right|<4 C^{\prime}\|Q\| d e^{-m a} ; \quad d=|\mathbf{x}|-r .
$$

Since $\|Q\|<1$ we get the uniform estimate (53) for the case of $f^{(1)}$. 
The second statement is most easily verified by writting the operator $K_{E, R}$ as an integral kernel $K\left(\mathrm{x}_{1} \ldots \mathrm{x}_{n} ; \mathrm{x}_{1}^{\prime} \ldots \mathrm{x}_{n}^{\prime}\right)$ and showing that it is of Hilbert-Schmidt type i.e.

$$
\int|K|^{2} d^{3} x_{i} d^{3} x_{i}^{\prime}<\infty .
$$

With these two results the compactness of the set (42) can be demonstrated in the following way. Choosing an arbitrary $\varepsilon>0$ we want to show that a family of vectors $\Psi_{i}$ in the set such that

$$
\left\|\Psi_{i}-\Psi_{j}\right\|>\varepsilon
$$

can only have a finite number of members. In other words, we want to show that it is impossible to choose an infinite number of $Q_{i}$ satisfying (49) such that

$$
\| P_{E}\left(Q_{i}-Q_{j}\right)|0\rangle \|>\varepsilon .
$$

Note that the expansion (50) of the vectors $P_{E} Q_{i}|0\rangle$ terminates at $n \approx E / m$ due to the energy projection. Making the decomposition (51) we have

$$
P_{E} Q_{i}|0\rangle=K_{E, R} \Phi_{i}+X_{i}
$$

where $\left\|\Phi_{i}\right\| \leqq 1$ and $\left\|X_{i}\right\|<A R^{2} e^{-m R}$. Choosing $R$ big enough so that

equation (61) demands

$$
A R^{2} e^{-m R}<\frac{\varepsilon}{4}
$$

$$
\left\|K_{E, R}\left(\Phi_{i}-\Phi_{j}\right)\right\|>\frac{\varepsilon}{2} .
$$

Since $K$ is a compact operator and $\left\|\Phi_{i}\right\| \leqq 1$ there can be only finite families of vectors $\Phi_{i}$ satisfying (64).

\section{b) Generalized Free Field}

A generalized free field is characterized by its Lehmann weight $\varrho\left(\varkappa^{2}\right)$. We treat here the case where $\varrho$ is a smooth function with support in a finite interval $\varkappa_{1}<x<\varkappa_{2}$. Symbolically the field $A(x)$ can be written as

$$
A(x)=\int \varrho^{1 / 2}\left(\varkappa^{2}\right) A\left(x ; x^{2}\right) d \varkappa^{2}
$$

where $A\left(x ; x^{2}\right)$ has the commutation relations and equations of motion of an ordinary free field with mass $x$ :

$$
\begin{gathered}
{\left[A\left(x ; x^{2}\right), A\left(y ; x^{\prime 2}\right)\right]=\delta\left(x^{2}-x^{\prime 2}\right) \Delta(x-y, x),} \\
\left(\square-x^{2}\right) A\left(x ; x^{2}\right)=0 .
\end{gathered}
$$

As in the case of the free field $A(x)$ can be decomposed into

$$
A(x)=A^{(+)}(x)+A^{(-)}(x)
$$

where $A^{(+)}$is the creation part (negative frequency part, ) $A^{(-)}$the destruction part (positive frequency part). Correspondingly the Hilbert space can be decomposed into a direct sum of orthogonal subspaces 
$\mathscr{H}^{(k)}$ where $\mathscr{H}^{(k)}$ results from the application of $k$ creation operators $A^{(+)}$ on the vacuum. $\mathscr{H}^{(k)}$ may be called the $k$-quantum part of the Hilbert space.

We choose now one fixed test function $f(x)$ with support in a finite region $\mathcal{O}$ and a set of polynomials $P_{n}$ of one variable (to be specified below) and consider the sequence of operators $\star$ [with $f(x)$ and $P_{n}$ real]

$$
B_{n}=\int A(x)\left(P_{n}(\square) f\right) d^{4} x .
$$

Clearly the operator $Q_{n}=e^{i B_{n}}$ has norm one and belongs to $R(\mathcal{O})$. Since

$$
e^{i B_{n}}|0\rangle=e^{-1 / 2\left\langle 0\left|B_{n}^{2}\right| 0\right\rangle} e^{i B_{n}^{(+)}|0\rangle}
$$

we find that the one-quantum component of $Q_{n}|0\rangle$ is given by

$$
\Psi_{n}=i e^{-1 / 2\left\langle 0\left|B_{n}^{2}\right| 0\right\rangle} B_{n}|0\rangle \text {. }
$$

We can choose the polynomials $P_{n}$ so that

$$
\left\langle 0\left|B_{n} B_{m}\right| 0\right\rangle=\delta_{n m}
$$

i.e. that the $\Psi_{n}$ are mutually orthogonal and of equal length. This requires that we choose the $P_{n}$ as orthogonal polynomials with respect to the measure

with

$$
\varrho^{\prime}\left(x^{2}\right)=\varrho\left(x^{2}\right) \sigma\left(x^{2}\right)
$$

In other words we define the $P_{n}$ by

$$
\int P_{n}\left(\varkappa^{2}\right) P_{m}\left(\varkappa^{2}\right) \varrho^{\prime}\left(\varkappa^{2}\right) d \varkappa^{2}=\delta_{n m} .
$$

If $P_{E}$ is an energy projection, the family of vectors

$$
\Phi_{n}=P_{E} Q_{n}|0\rangle
$$

will be in the set (42) and since $P_{E}$ transforms each $n$-quantum subspace into itself we have

$$
\left\|\Phi_{n}-\Phi_{m}\right\|>\left\|P_{E}\left(\Psi_{n}-\Psi_{m}\right)\right\|=\Delta_{n m} .
$$

We want to show now that $\Delta_{n m}$ is larger than some finite positive $\varepsilon$ for all pairs $n, m$. One has

with

$$
\Delta_{n m}=e^{-1} \int \varrho\left(\varkappa^{2}\right) \sigma^{\prime}\left(\varkappa^{2}\right)\left(P_{n}-P_{m}\right)^{2} d \varkappa^{2}
$$

$$
\sigma^{\prime}\left(\varkappa^{2}\right)=\int_{\left(p^{2}+\varkappa^{2}\right)<E^{2}}\left(\mathbf{p}^{2}+\varkappa^{2}\right)^{-1 / 2}\left|\tilde{f}\left(\mathbf{p},\left(\mathbf{p}^{2}+\varkappa^{2}\right)^{1 / 2}\right)\right|^{2} d^{3} p .
$$

Comparing (77) and (72) we see that one can find a positive constant $C$ such that

$$
\sigma^{\prime}\left(\varkappa^{2}\right)>C \sigma\left(\varkappa^{2}\right) \text { for } x_{1}^{2}<\varkappa^{2}<E^{2}-\lambda^{2} .
$$

\footnotetext{
* This sequence was suggested to us by D. W. Robinson.
} 
The constant $C$ depends of course on $\lambda$ and goes to zero for $\lambda \rightarrow 0$. Choosing for the moment $E>\varkappa_{2}$ one finds

$$
\Delta_{n m}>e^{-1} C \int \varrho^{\prime}\left(x^{2}\right)\left|P_{n}-P_{m}\right|^{2} d x^{2}>2 e^{-1} C .
$$

Thus for $E>\varkappa_{2}$ one can find an infinite family of vectors in the set (42) for which $\Delta_{n m}>C^{\prime}>0$. In other words the set is not compact in this model. The choise of $E>\varkappa_{2}$ was made to simplify the proof. Our result still holds for $E<\varkappa_{2}$ since one can approach $\Phi_{n}=e^{i D_{n}}|0\rangle$ with

$$
D_{n}=\int_{x_{1}^{2}}^{x_{2}^{2}-\lambda^{2}} \varrho^{1 / 2}\left(x^{2}\right) A\left(x, x^{2}\right) P_{n}^{\prime}(\square) f(x) d^{4} x d x^{2}
$$

by

$$
X_{n}=\exp i \sum_{k=0}^{N(n, \varepsilon)} a_{k} B_{k}|0\rangle
$$

with arbitrary accuracy $\varepsilon$

$$
\left\|\Phi_{n}-X_{n}\right\| \leqq \varepsilon
$$

and therefore one could have started from $\Phi_{n}$ instead of $Q_{n}|0\rangle$ in our previous proof. Thus one would have found $\left\|P_{E}\left(\Phi_{n}-\Phi_{m}\right)\right\|>C$ as long as $E>\varkappa_{2}^{2}-\lambda^{2}$ and choosing $\varepsilon=C / 4$

$$
\left\|P_{E}\left(X_{n}-X_{m}\right)\right\|>C / 2
$$

which shows that the set (42) is not compact for arbitrary $E$ in this model.

Acknowledgements. Originally this work started in collaboration with D. W. RoBINson who has contributed several valuable ideas in particular to Section IV. We have also profited from discussions with D. KASTLER, M. ZERNER and S. SCHLIEDER. Finally we would like to thank the Physics Division of the Aspen Institute for Humanistic Studies for their hospitality during the final stages of this work and the National Science Foundation for support.

\section{References}

[1] Greenberg, O. W.: Annals of Phys. 10, 158 (1961).

[2] HaAg, R., and B. Schroer: J. Math. Phys. 3, 248 (1962).

[3] Bonchers, H. J.: Three Remarks on Quantum Field Theory, N.Y.U. progress report 1963.

[4] AraKi, H.: Lecture notes. Zürich 1963.

[5] ReEH, H., and S. Schlieder: Nuovo Cimento 22, 1051 (1961).

[6] Knight, J. M.: J. Math. Phys. 2, 459 (1961).

[7] Ruelle, D.: Helv. Phys. Acta 35, 147 (1962).

[8] Schlieder, S.: Commun. Math. Phys. 1, 265 (1965).

[9] Kolmogoroff, A. N.: Doklady Akad. Nauk SSSR 120, 239 (1958).

[10] Gelfand, I. M., and N. J. Wilenkin: Verallgemeinerte Funktionen. Vol. IV, p. 96. Berlin: Deutscher Verlag der Wissenschaften 1964. 